\title{
3D Hydrogels Containing Interconnected Microchannels of Subcellular Size for Capturing Human Pathogenic Acanthamoeba Castellanii
}

Sören B. Gutekunst, ${ }^{\dagger}$ Katharina Siemsen, ${ }^{\dagger}$ Steven Huth, ${ }^{\dagger}$ Anneke Möhring, ${ }^{\dagger}$ Britta Hesseler, ${ }^{\dagger}$ Michael Timmermann, ${ }^{\dagger}$ Ingo Paulowicz, ${ }^{\S}$ Yogendra Kumar Mishra, ${ }^{\ddagger}$ (i) Leonard Siebert, ${ }^{\dagger}$ Rainer Adelung, and Christine Selhuber-Unkel* ${ }^{\ddagger} \dagger$ (1)

\begin{abstract}
${ }^{\dagger}$ Institute for Materials Science, Biocompatible Nanomaterials, and ${ }^{\ddagger}$ Institute for Materials Science, Functional Nanomaterials, University of Kiel, Kiel D-24143, Germany

${ }^{\S}$ Phi-Stone AG, Kiel D-24143, Germany
\end{abstract}

Supporting Information

ABSTRACT: Porous hydrogel scaffolds are ideal candidates for mimicking cellular microenvironments, regarding both structural and mechanical aspects. We present a novel strategy to use uniquely designed ceramic networks as templates for generating hydrogels with a network of interconnected pores in the form of microchannels. The advantages of this new approach are the high and guaranteed interconnectivity of the microchannels, as well as the possibility to produce channels with diameters smaller than $7 \mu \mathrm{m}$. Neither of these assets can

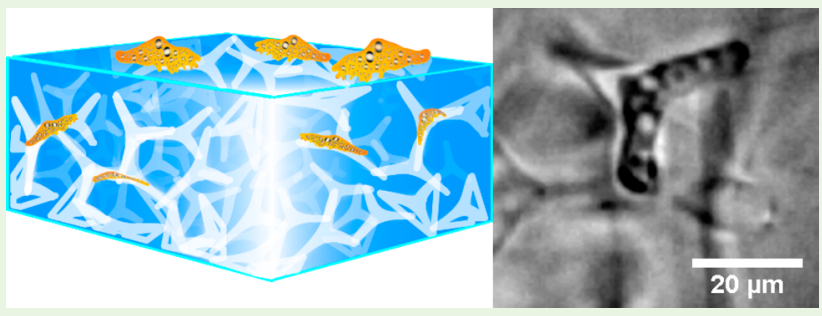
be ensured with other established techniques. Experiments using the polyacrylamide substrates produced with our approach have shown that the migration of human pathogenic Acanthamoeba castellanii trophozoites is manipulated by the microchannel structure in the hydrogels. The parasites can even be captured inside the microchannel network and removed from their incubation medium by the porous polyacrylamide, indicating the huge potential of our new technique for medical, pharmaceutical, and tissue engineering applications.

KEYWORDS: 3D ceramic templates, microchannels, porous hydrogel, cell migration, human pathogens

\section{INTRODUCTION}

The significant influence of the extracellular environment on a variety of cellular processes such as cell adhesion, proliferation, migration and differentiation has recently developed into a subject of high interest for cellular research. ${ }^{1-3}$ Cell migration, for instance, is controlled by the dimension ${ }^{4,5}$ as well as by the mechanical properties of the extracellular environment, ${ }^{6,7}$ where nuclear deformability, physical tissue constraints, cell stiffness, and cell adhesion are important limiting factors. ${ }^{8-10}$ Therefore, the manipulation and characterization of microstructural properties of materials provides novel opportunities to mimic extracellular environments in order to control cell migration.

A biomedically highly relevant application for controlling cell migration is to capture pathogenic microorganisms. For example, micron-sized lobster traps have been fabricated from silicone to capture swimming bacteria. ${ }^{11}$ Such structure-based capture devices are very interesting, as they can provide cell capture without the requirement of additional chemical agents.

The medical advantages of cell-capturing materials become quickly apparent considering pathogenic cells that rely on adhesion to their surroundings. A medically dangerous, ${ }^{12}$ adhesive, ${ }^{13}$ and extremely motile ${ }^{14}$ example of such pathogens is Acanthamoeba castellanii (A. castellanii). Upon contact with the human eye, this amoeba invades the corneal tissue and leads to a painful and hard-to-treat Acanthamoeba keratitis, which can even cause blindness. ${ }^{15}$ This is an especially troubling issue as $A$. castellanii is found in a huge variety of environments such as water reservoirs (e.g., swimming pools or liquids for contact lens storage $\left.^{16}\right)$ and soil, even despite disinfection procedures. ${ }^{17}$ Thus, the infection chance during everyday human life is comparably high. ${ }^{18}$ A. castellanii infections are especially severe as the parasite's motile trophozoite form can transform into double-walled cysts under unfavorable conditions to protect itself from medication, heat, or even radiation. This makes treatment procedures extremely long-lasting and complicated. ${ }^{19}$ Considering this, it is not only important to improve procedures that cure an A. castellanii infection, but also to find methods to avoid the infection in the first place. Conventional strategies to avoid $A$. castellanii infections are based on disinfection procedures, ${ }^{20}$ which lack the efficiency to kill all the amoebae ${ }^{21}$ and strongly rely on the active cooperation of the potentially exposed person. Hence, they are prone to mistakes and

Received: August 24, 2018

Accepted: January 10, 2019

Published: January 10, 2019 
capturing the amoeba using microstructured materials poses a highly promising alternative to these established methods.

Here, we introduce a novel approach to capture $A$. castellanii by the structural features of a $3 \mathrm{D}$ porous material. To do so, we produced bulk hydrogels containing a maze-like three-dimensional network of interconnected microchannels by embedding and subsequently dissolving microfibrous zinc oxide $(\mathrm{ZnO})$ scaffolds in Polyacrylamide (PAAm). The scaffolds are made of $\mathrm{ZnO}$ tetrapods, which are very unique three-dimensional structures with four arms interconnected via a central core at an angle of $\sim 109^{\circ}$ and with variable sizes in the micrometer regime. ${ }^{22}$ By pressing such tetrapods into a tablet and sintering them at high temperatures, a highly interconnected $\mathrm{ZnO}$ network can be produced that serves as sacrificial structure for microfibrous materials. ${ }^{23,24}$ When employed in combination with hydrogels, a unique microchannel network is formed that can be used both for controlling A. castellanii migration and even for capturing these parasites from their incubation medium. Our findings indicate the high potential of this approach to lower the risk of $A$. castellani infections on a broad scale, as it can be used to produce materials to remove the parasites from water reservoirs or to inhibit amoebae migration, giving our approach a high medical, pharmaceutical, and engineering relevance.

\section{MATERIALS AND METHODS}

Acanthamoeba Culture. Acanthamoeba castellanii trophozoites were cultured at room temperature in peptone yeast glucose (PYG) 712 medium (20 g proteose peptone (BD, Sparks, USA), $1 \mathrm{~g}$ of yeast extract (BD, Sparks, USA), $950 \mathrm{~mL}$ of distilled water, $10 \mathrm{~mL}$ of $0.4 \mathrm{M} \mathrm{MgSO}_{4}$. $7 \mathrm{H}_{2} \mathrm{O}$ (AppliChem, Darmstadt, Germany), $8 \mathrm{~mL}$ of $0.05 \mathrm{M} \mathrm{CaCl}_{2}$ (AppliChem, Darmstadt, Germany), $34 \mathrm{~mL}$ of $0.1 \mathrm{M}$ sodium citrate$2 \mathrm{H}_{2} \mathrm{O}$ (Merck, Darmstadt, Germany), $10 \mathrm{~mL}$ of $0.005 \mathrm{M} \mathrm{Fe}\left(\mathrm{NH}_{4}\right)$ $2\left(\mathrm{SO}_{4}\right)_{2} \cdot 6 \mathrm{H}_{2} \mathrm{O}$ (AppliChem, Darmstadt, Germany), $10 \mathrm{~mL}$ of $0.25 \mathrm{M}$ $\mathrm{Na}_{2} \mathrm{HPO}_{4} \cdot 7 \mathrm{H}_{2} \mathrm{O}$ (Roth, Karlsruhe, Germany), $10 \mathrm{~mL}$ of $0.25 \mathrm{M}$ $\mathrm{KH}_{2} \mathrm{PO}_{4}$ (Roth, Karlsruhe, Germany), and $50 \mathrm{~mL}$ of $2 \mathrm{M}$ glucose (Sigma-Aldrich Chemie GmbH, Steinheim, Germany)). The PYG medium was exchanged at least once a week to avoid cyst formation. Acanthamoebae were detached from the culture flask by slight knocking, collected with a pipet and centrifuged. The generated pellet was resuspended in PYG medium and the cell number was counted using a Neubauer counting chamber.

Ceramic Porous Template Synthesis. Zinc oxide tetrapods ( $t$ $\mathrm{ZnO}$ ) were synthesized by using a single step flame transport synthesis approach. $^{22,23}$ The tetrapods with arm diameter in the submicron regime and arm lengths ranging between 1 and $8 \mu \mathrm{m}$ were utilized for fabrication of porous interconnected sacrificial templates for all cellular experiments. Thinner tetrapods for checking the influence of tetrapod diameter onto the resulting channel diameter were produced by adapting the flame transport synthesis: Whereas the conventional synthesis of the tetrapods presented above makes use of a sacrificial polymer (PVB) as a carbon source for zinc reduction, we now used ethanol in its stead. Furthermore, a turbulent air environment was created by applying a constant pressurized air flow of $90 \mathrm{~L} / \mathrm{min}$. Additional turbulent effects from ethanol combustion as well as a shortterm temperature increase led to a faster growth of thinner and longer tetrapod arms, partly connected by nanosails. ${ }^{25}$

To form the 3D sacrificial templates, tetrapods from one synthesis batch were filled and pressed into cylindrical alumina molds and the amount was chosen such that the density of the resulting network is 0.3 , $\sim 0.49,0.6$, or $1.0 \mathrm{~g} / \mathrm{cm}^{3}$. The templates with pressed $\mathrm{ZnO}$ tetrapods were annealed at high temperature (thick tetrapods at $1150{ }^{\circ} \mathrm{C}$ for 4.5 $\mathrm{h}$; thin tetrapods at $800^{\circ} \mathrm{C}$ for $5 \mathrm{~h}$ ) to form interconnections. For all cell experiments, we employed scaffolds with a density of $0.49 \mathrm{~g} / \mathrm{cm}^{3}$.

Template-Mediated Polymerization of Microchannel-Containing Polyacrylamide. A mixture of acrylamide (Bio-Rad, 40\%, $1.00 \mathrm{~mL}$ ), $N, N^{\prime}$-methylenebis(acrylamide) (Bis, Bio-Rad, 2\%, $200 \mu \mathrm{L}$ ), HEPES buffer (Sigma-Aldrich, pH 7.5, $50.0 \mu \mathrm{L}$ ), and ammonium persulfate solution (Sigma-Aldrich, $10 \%$, aq., $75.0 \mu \mathrm{L}$ ) were filled up to a volume of $5.00 \mathrm{~mL}$ with bidest. water in a small beaker and degassed for $20 \mathrm{~min}$ in a desiccator. The solution was poured on the $t-\mathrm{ZnO}$ tablet with $N, N, N^{\prime}, N^{\prime}$-tetramethylethylenediamine (TEMED, Bio-Rad, 5.00 $\mu \mathrm{L}$ ), and after $1 \mathrm{~h}$ of polymerization, the substrate was washed with and stored in bidest. water (AppliChem GmbH).

Hydrolysis of the ZnO-Template inside the Polyacrylamide. The $\mathrm{ZnO}$-template was removed from the PAAm via hydrolyzation by three times incubating the hydrogel in hydrochloric acid ( $0.5 \mathrm{M}$, SigmaAldrich) for a total of at least $24 \mathrm{~h}$. Afterward, the hydrogel was washed with bidest. water (AppliChem $\mathrm{GmbH}$ ) until it had a neutral $\mathrm{pH}$ value (pH 6 to 7 ) and was swollen to equilibrium in an adenosine $3^{\prime}, 5^{\prime}$-cyclic monophosphate solution (cAMP, Sigma-Aldrich, 0.01-10.0 mM), which was exchanged daily, for 2 to $4 \mathrm{~d}$. Samples were sterilized in $70 \%$ ethanol for $15 \mathrm{~min}$ and washed under sterile conditions for $24 \mathrm{~h}$ with PYG 712 for A. castellanii experiments. Substrates were used within 48 h.

Fluorescent Staining of the Microchannels. To render the microchannels of a PAAm sample fluorescent, we removed the water from the hydrogel by washing it repeatedly in ethanol (Walter CMP, Germany) for at least $20 \mathrm{~min}$, increasing the ethanol concentration with each washing step. The concentrations employed were 50, 70, 80, 90, 95 , and $99 \%$. Then, the samples were incubated in an aqueous solution of Fluorescein isothiocyanate - Dextran 500.000 - Conjugate (FITCDextran, $1.32 \mathrm{mg} / \mathrm{mL}$, Sigma-Aldrich) overnight.

Imaging of the Microchannels. Fluorescent z-stack images were recorded using a confocal microscope (Olympus, IX-81), equipped with a spinning disc unit (TILL Photonics, 1203-9-1-0017), a 488 nm Laser (Topica Photonics, iCHROME-MLE_LFA 3002) and an EM-CCD Digital Camera (Hamamatsu). The samples were placed upside down in glass-bottom Petri dishes (ibidi, 81218-200). The surface at the bottom of the sample was focused, and the focus plane was shifted into the sample up to a point where background fluorescence became too strong ( $\sim 70 \mu \mathrm{m}-220 \mu \mathrm{m}$ into the sample). Z-stacks where recorded between these two focus planes with a step width of $1.99 \mu \mathrm{m}$. The z-stack top-views were computed using the xcellence rt software (Olympus, version 1.2).

Cell Migration Experiments. The sterilized microchannelcontaining PAAm samples were incubated with $A$. castellanii (ATTC 30234, 30.000 cells $/ \mathrm{mL}$ ) in PYG medium in a 6-well plate (Sarstedt). After $0.5 \mathrm{~h}-2 \mathrm{~h}$ incubation time, phase contrast images (Olympus, IX81/BX-43) and movies ( 1 frame per $5 \mathrm{~s}$ ) of the trophozoites inside the microchannels were recorded with a $10 \times$ objective using a monochrome (Hamamatsu, C-9300) or color camera (Imaging Source, DFK 31BF03). As control experiments, bulk PAAm samples were created as described above, but without applying a $\mathrm{ZnO}$ template, and swollen to equilibrium in cAMP.

Image Analysis. Acanthamoeboa images were analyzed for the resting time, cell speed, and the direction of movement of the trophozoites inside the channels. Cell speed was determined by following the track of their front and rear inside a channel. The speed of the moving amoebae and their diameter perpendicular to the direction of motion was measured using Image J. ${ }^{26}$ The latter was interpreted as a measure for the channel diameter the cell was migrating through.

Cell Trapping Efficiency of the Microchannel-Containing Polyacrylamide Substrates. To quantify the efficiency with which our microstructured PAAm captures Acanthamoeba from incubation medium, we placed four PAAm substrates in one well of a 6-well-plate and incubated them with A. castellanii in PYG 712 medium (20000 cells per well). These experiments were performed in three technical repeats and also with blank wells as control. The number of cells in the supernatant of each well was counted with a Hemocytometer (Neubauer counting chamber, Hecht Glaswaren) after 2, 24, 48, and $120 \mathrm{~h}$.

Elasticity of Bulk Samples. Bulk samples without $\mathrm{ZnO}$ were prepared according to the prescription above. The polymer mixture was poured in PTFE molds with a diameter of 10 and $5 \mathrm{~mm}$ height. The samples were polymerized at room temperature for $1 \mathrm{~h}$ and then treated with either dest. water, $0.5 \mathrm{M}$ hydrochloric acid or $1.28 \mathrm{M}$ hydrochloric acid for 2 days. Afterward, the samples were washed with dest. water 

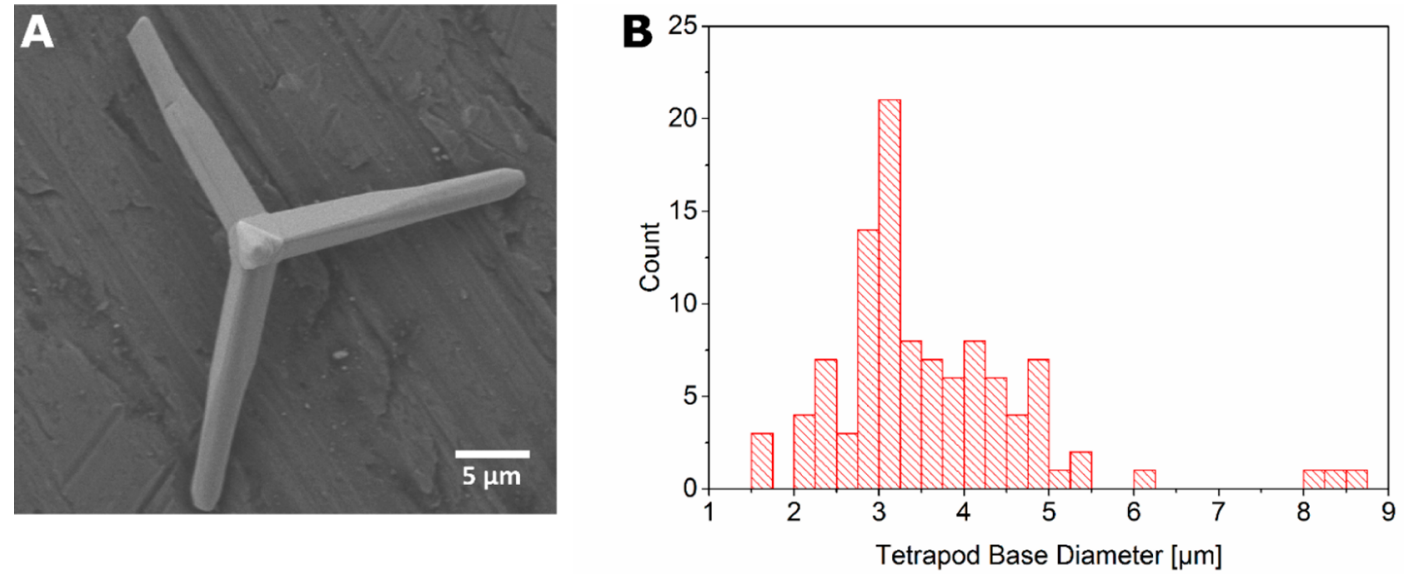

Figure 1. (A) Exemplary SEM image of a $\mathrm{ZnO}$ tetrapod, which serves as basic constituent of sacrificial templates for the production of microchannelcontaining hydrogels. The SEM images allow the determination of the tetrapod size by image analysis. A histogram of the tetrapod diameter, measured at the base of the tetrapod arms is shown in B. The distribution ranges from 1.5 to $8.75 \mu \mathrm{m}$ and peaks at $3 \mu \mathrm{m}$.

until the $\mathrm{pH}$ of the solution was neutral (up to $6 \mathrm{~d}$ ). The mechanical properties of the swollen samples were determined with an indentation experiment using a modified tensile test setup. On one side, a stepper motor (M229.26S, Physik Instrumente GmbH \& Co. KG, Germany) introduces a movement and on the other side, a load cell (KD24s $10 \mathrm{~N}$, ME-Meßsysteme GmbH, Germany) detects a force. To switch this setup to a tensile test setup, we mounted a PTFE-sphere with a diameter of $6 \mathrm{~mm}$ to the moving arm of the stepper motor and a flat PTFE plate to the load cell. PTFE was chosen for reducing the friction between the surfaces and the sample. For the measurement, the sphere was moved to a predefined position above the sample. Afterward, it was lowered by 3 $\mathrm{mm}$ with a velocity of $0.1 \mathrm{~mm} / \mathrm{s}$, resulting in an indentation of the sample. The indentation depth varied between the samples, because the start- and end point of the measurement were predefined and the sample thickness varied.

\section{RESULTS}

We synthesized $\mathrm{ZnO}$ tetrapods, pressed them into a tablet, and sintered this tablet to ensure interconnectivity of the tetrapods in the resulting $\mathrm{ZnO}$ network. Figure 1A shows an exemplary Scanning Electron Microscopy (SEM) image of a $\mathrm{ZnO}$ tetrapod, the size of which can be easily determined by image analysis. Figure 1B shows a histogram of the diameters of 105 tetrapod arms, measured at their base. These diameters range from 1.5 to $8.75 \mu \mathrm{m}$ and their distribution peaks at $3 \mu \mathrm{m}$.

The $\mathrm{ZnO}$ tetrapod tablet was immersed in a solution of Acrylamide and BIS Acrylamide, which was then polymerized to form PAAm. A phase contrast image of the tetrapod tablet, embedded in the polyacrylamide gel, is shown in Figure 2A. When such a $\mathrm{ZnO}$-containing PAAm sample was incubated in hydrochloric acid, the $\mathrm{ZnO}$ template dissolved, leaving microchannels in the hydrogel. In Figure 2B, the dissolving process is shown macroscopically and its final stages are captured microscopically (Figure 2C) at the center of the original tablet, showing that no $\mathrm{ZnO}$ tetrapods were left after the hydrolysis. The sample was then washed excessively in aqua bidest. to remove all the dissolved $\mathrm{ZnO}$ and other reaction products, thus resulting in microchannels inside the PAAm (Figure 2D). As the tetrapods had been pressed to high densities and were sintered at high temperatures prior to embedding them in the hydrogel, the $\mathrm{ZnO}$ network in the PAAm was fully interconnected and hence the microchannels, which remained in the hydrogel as a negative imprint of this $\mathrm{ZnO}$ network after its dissolution, were also interconnected. In other words, the hydrogel now contained a network of cylindrically shaped, interconnected microchannels with larger cavities at the junctions of several of these channels. Figure 3 visualizes the different fabrication steps of our procedure.

For better visualization of the channels, we filled them with a FITC-Dextran conjugate (which entered the microchannels, but not the hydrogel itself), and imaged z-stacks of the samples with a confocal microscope. Exemplary top-views of z-stack composites are presented in Figure 4. For each of two different tetrapod diameter ranges ( $\mathrm{A}-\mathrm{C}$ and $\mathrm{D}-\mathrm{F}$, respectively), three different samples with different tetrapod densities $\left(0.3 \mathrm{~g} / \mathrm{cm}^{3}\right.$ in $\mathrm{A}$ and $\mathrm{D}, 0.6 \mathrm{~g} / \mathrm{cm}^{3}$ in $\mathrm{B}$ and $\mathrm{E}, 1 \mathrm{~g} / \mathrm{cm}^{3}$ in $\mathrm{C}$ and $\mathrm{F}$ ) were produced and for each sample, one image is given. The images show that the densities as well as diameter ranges of the resulting microchannels can be controlled by manipulating the respective property of the sacrificial scaffold. The density can be varied by adapting the amount of $\mathrm{ZnO}$ tetrapods added to the sample as well as by changing the pressure applied during the sintering process. More tetrapods as well as higher pressures increase the network density. The diameter ranges of the tetrapods and hence the microchannels can be varied by parameters of the tetrapod production process. ${ }^{27}$

To investigate the impact of our 3D hydrogel microstructure on the migration of Acanthamoebae, we incubated our microchannel-containing PAAm with A. castellanii trophozoites, which readily adhered to the PAAm without further surface functionalization. As A. castellanii is responsive to adenosine $3^{\prime}, 5^{\prime}$-cyclic monophosphate solution (cAMP), ${ }^{28}$ the hydrogels were soaked in cAMP prior to cell experiments to accelerate migration into the hydrogel scaffold. Two very interesting and striking effects became quickly apparent: First, in spite of their typical diameter of $12 \mu \mathrm{m}-35 \mu \mathrm{m},{ }^{12}$ A. castellanii trophozoites were able to migrate through microchannels with diameters down to a few microns to explore the material (Figure 5A). Second, the trophozoites often remained immotile for much longer times when reaching larger cavities at the junctions of the microchannels inside the PAAm (Figure 5B).

To further quantify these effects, movies were taken over several hours (here, $16 \mathrm{~h}$; Movie S1) and analyzed for cell migration speed, cell diameter (measured perpendicular to direction of movement, hence corresponding to the size of the microchannel that the amoeba was squeezing through) as well as the percentage of migrating and resting $A$. castellanii 
A

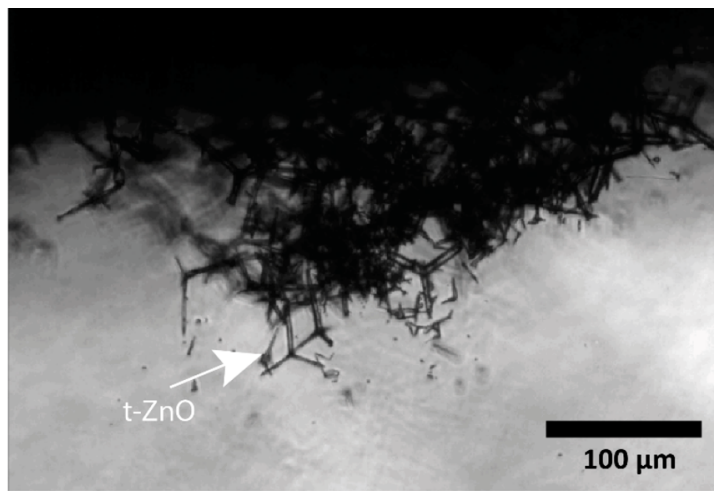

B
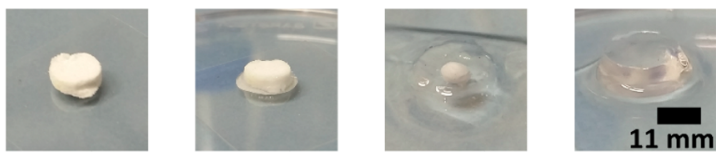

C

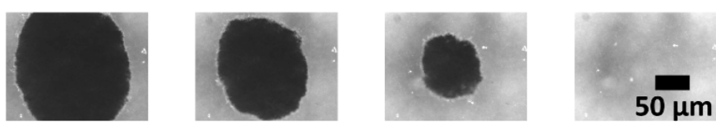

D

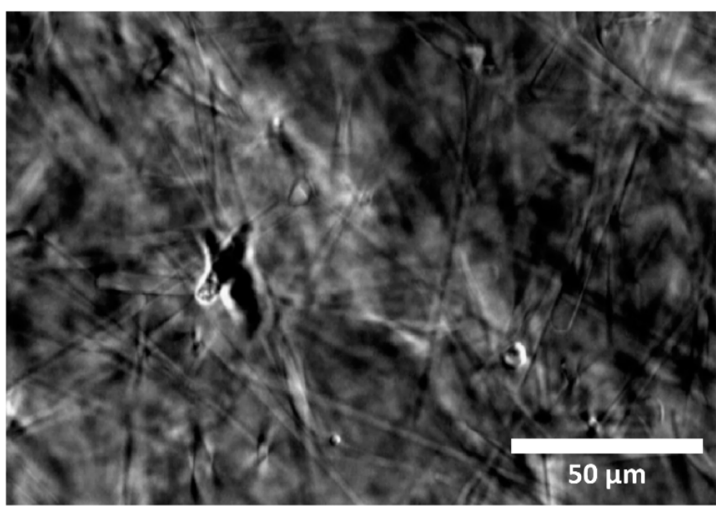

Figure 2. $\mathrm{ZnO}$ tetrapods are pressed and sintered into an interconnected $\mathrm{ZnO}$ network and embedded into an Acrylamide solution, which is then allowed to polymerize. (A) Phase contrast image of the $\mathrm{ZnO}$ network in the PAAm hydrogel. The $\mathrm{ZnO}$ network dissolves when the PAAm sample is incubated in hydrochloric acid $(\mathrm{HCl})$. (B, C) both present time arrays starting from the left that recorded the dissolution process. (B) Entire process shown macroscopically. (C) Phase contrast images of the last stages of the hydrolysis, recorded microscopically every $15 \mathrm{~min}$ at the center of the tablet, showing that the nontransparent $\mathrm{ZnO}$ structures dissolve completely. The $\mathrm{ZnO}$ structures leave an interconnected network of microchannels in the PAAm gel, as shown in a phase contrast image in $\mathrm{D}$.

trophozoites. As a control, we carried out the same analysis on $A$. castellanii migrating on bulk PAAm samples. The results are presented in Figure 6 and demonstrate that A. castellanii trophozoites squeezed into channels of diameters down to 6.5 $\mu \mathrm{m}$. No migrating cells were observed in channels smaller than this. Furthermore, no apparent correlation of cell migration speed with the channel diameter was observed, but $A$. castellanii migrating on bulk PAAm samples were able to reach velocities almost twice as high as the ones in the microchannel networks (Figure 6A). Even more strikingly, we found that more than 50\% of the A. castellanii trophozoites in microchannel-containing PAAm were resting in cavities while amoeba migrating on bulk PAAm samples were always in motion (Figure 6B), underlining the significance of the influence that our new microchannel

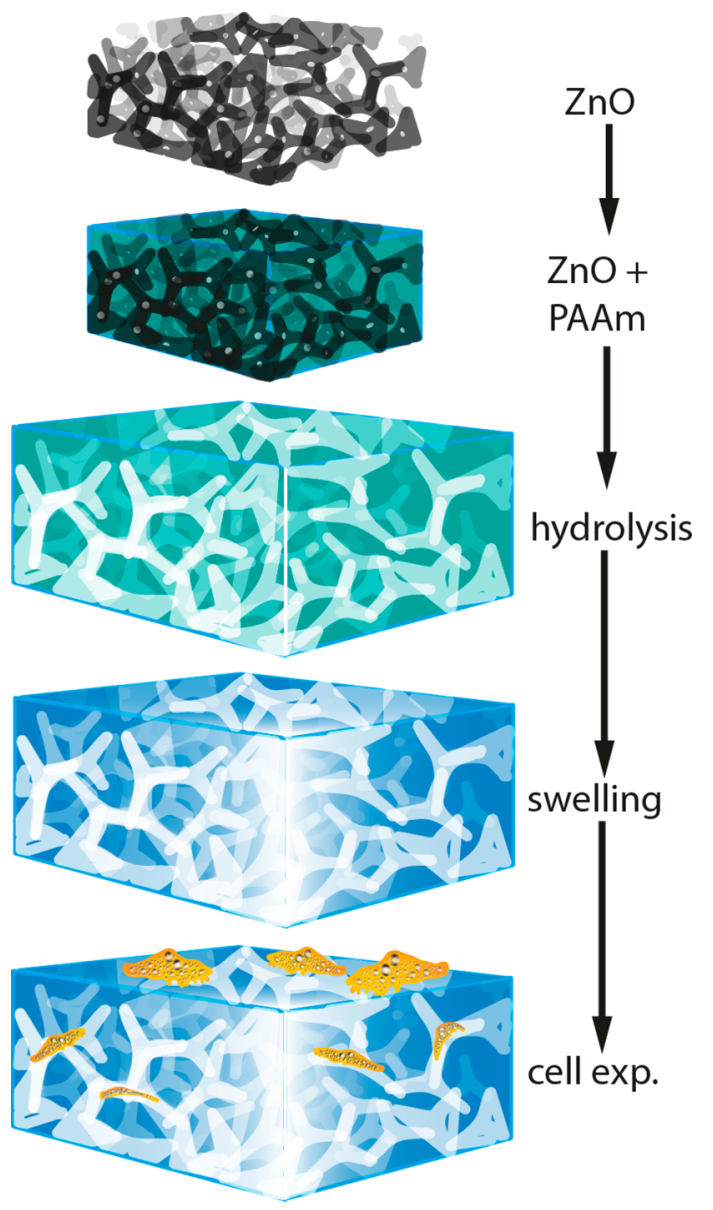

Figure 3. Synthesis of microchannel-containing polyacrylamide hydrogels. First, a sintered zinc oxide tetrapod based network template (black) with a well-defined tetrapod density is embedded into an acrylamide solution, which is then polymerized to polyacrylamide (PAAm, green). Afterward, the template is hydrolyzed with hydrochloric acid (HCL), leaving a negative of microchannels inside the PAAm (white). After complete hydrolysis of the ceramic template, the PAAm is washed and swollen to equilibrium in cAMP solution. Now, the material can be used for cell experiments (orange). As the tetrapod template was sintered and produced at high tetrapod densities, the network of microchannels in the PAAm, being a negative of this template, is highly interconnected.

network in the PAAm gels has on cell migration. An Acanthamoeba was defined as "resting" if it stayed inside a cavity for at least $40 \mathrm{~s}$. This value was chosen heuristically due to the length of our movies. In some experiments, the trophozoites stayed inside a cavity even for several hours. It is noteworthy that A. castellanii trophozoites are very motile and clearly do not rest on flat, unstructured substrates.

Experiments carried out at longer time scales demonstrated that $A$. castellanii trophozoites stayed in the microchannel network inside our PAAm substrate for at least 6 days. They continued to migrate and exhibit intracellular motion while no encystment but very high trophozoite densities were observed in the channels (Figure 7). These rising cell densities motivated the idea that the specific 3D architecture of microchannels in combination with larger cavities in our hydrogels might provide the opportunity to capture $A$. castellanii from liquids with our new material. Hence, we incubated microchannel-containing PAAm samples up to $120 \mathrm{~h}$ with A. castellanii in PYG medium and repeatedly counted the number of cells in the supernatant of 

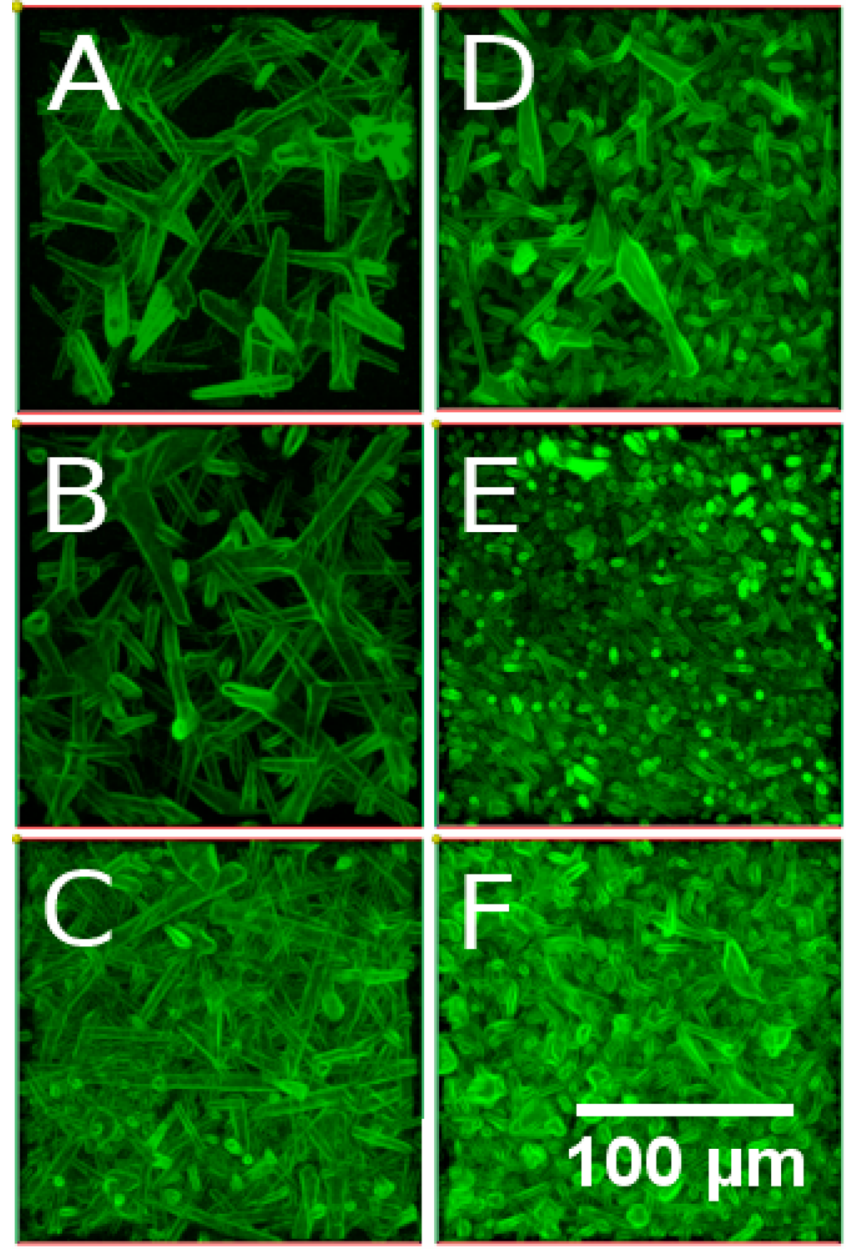

Figure 4. Top-view of a z-stack of confocal fluorescence microscopy images of the microchannels in PAAm hydrogels. The images present channels resulting from tetrapod scaffolds with different network densities and diameters. (A-C) Samples produced by using tetrapods with larger diameters, (D-F) samples produced using thinner tetrapods. Each column of images pictures samples from one $\mathrm{ZnO}$ density in the tetrapod template. Namely (A, D) 0.3, (B, E) 0.6, and (C, F) $1 \mathrm{~g} / \mathrm{cm}^{3}$. It becomes clear that the ranges of channel diameters and densities are influenced by these respective parameters of the sacrificial $\mathrm{ZnO}$ template and can hence be controlled by adapting the template properties.

the medium (Figure 8). As a control, we repeated these experiments in cell culture dishes without PAAm. After 24 and $48 \mathrm{~h}$ incubation time, the amount of Acanthamoeba was slightly higher in the medium of the microchannel-containing PAAm, but after $120 \mathrm{~h}$ the presence of the microporous material had led to a significantly lower amount of $A$. castellanii in the supernatant compared to the medium of the bulk control (Figure 8).

\section{DISCUSSION}

We have introduced a new technique to produce microstructured $3 \mathrm{D}$ hydrogels as cell substrates by pressing and sintering $\mathrm{ZnO}$ tetrapods into an interconnected network, which is immersed in an Acrylamide solution prior to polymerization. When the solution has polymerized, the tetrapod network is removed from the resulting PAAm hydrogel by hydrolysis with $\mathrm{HCl}$. We have shown that this approach produces PAAm substrates containing a network of interconnected microchannels with diameters down to only few microns and that these porous materials can be used as $3 \mathrm{D}$ substrates to study and manipulate the migration behavior of $A$. castellanii trophozoites.

This, however, is just one among the many possible applications of these tetrapod-based materials in engineering and corresponding applications. In fact, following the strategy of material deposition and subsequent removal of an underlying template, 3D hollow tetrapodal networks with tunable dimensions (from nanometer to micrometer scale) and ultrahigh porosities (up to $98 \%$ ) can in principle be easily realized also using many other ceramic materials. For instance, the 3D ceramic templates have already been successfully produced from several oxides, nitrides, silcon, etc. Because zinc oxide is easily hydrolyzed by acids, ${ }^{24}$ it was chosen for the present experiments as the sacrificial template material. Furthermore, all polymers that withstand acidic environments can be microstructured. In other words, using our new approach, a huge variety of materials can be equipped with a network of interconnected microchannels with many different channel diameters and densities (i.e., pore sizes, pore distribution, and porosity).

We rendered the channels in the hydrogels fluorescent (Figure 4), enabling us to measure channel diameters and densities directly in the hydrogel samples. Furthermore, by analyzing confocal z-stack slices, it is possible to determine the number of junctions as well as their positions (Figure S3). This allows describing microchannel sizes and distributions in the sample (Figure S4). The porosity of the sample can also be determined using these z-stack slices. The porosity $\Phi$ is defined as the ratio of the volume of void space (i.e., of our channels) to the total sample volume.

The total channel volume directly after the $\mathrm{ZnO}$ dissolution process is equal to the total volume of the tetrapod network and can hence be calculated from the weight of the sacrificial $\mathrm{ZnO}$ template, as the density of $\mathrm{ZnO}$ is known. The porosity can then be expressed as ${ }^{29}$

$$
\Phi=\frac{V_{\mathrm{ZnO}}}{V_{\mathrm{PAAm}}+V_{\mathrm{ZnO}}}=1-\frac{V_{\mathrm{PAAm}}}{V_{\mathrm{PAAm}}+V_{\mathrm{ZnO}}}
$$

with $V_{\text {PAAm }}$ and $V_{\mathrm{ZnO}}$ as the volume of PAAm or $\mathrm{ZnO}$, respectively. Hence, the initial PAAm porosity can be increased by increasing the amount of $\mathrm{ZnO}$ tetrapods in the sacrificial template. However, as the hydrogel continues to swell after $\mathrm{ZnO}$ dissolution, ${ }^{30}$ and since the description in eq 1 does not take local variations of channel densities or diameters into account, it is recommendable to evaluate the microporosity at the region of interest using fluorescence images (Figure 4). Using image analysis, the fraction of fluorescent pixels to the total amount of pixels of each slice of the z-stack can be calculated and hence gives the local microporosity of the swollen gel.

In general, we suggest being careful with global descriptions of the samples produced with our approach because, as seen in Figure $1 \mathrm{~B}$, the tetrapods constructing the sacrificial network do not have one distinct diameter, but a distribution of diameters. The same holds for the density of the tetrapod network and indeed, different positions of the microchannel-containing PAAm samples display different channel densities. Nevertheless, the properties of the channel network can be tuned as displayed in Figure 4, but it is rather useful to consider this as an adaption of ranges of channel densities and diameters. Another important aspect is to realize that the shape of tetrapod arms (Figure 1A) results in channels that vary their diameter across channel length. 
A

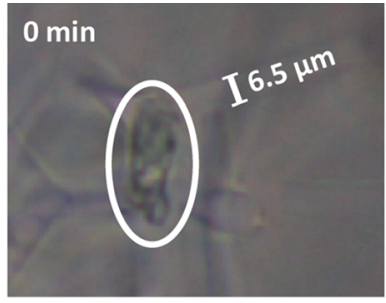

B

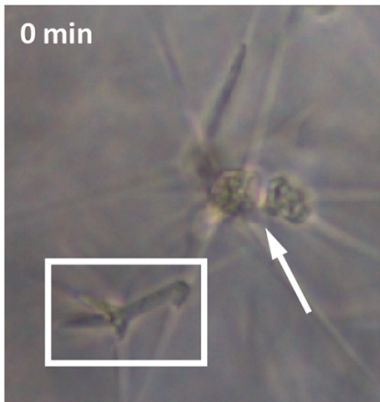

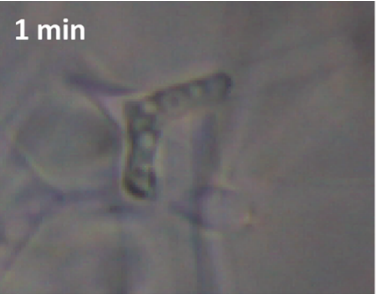
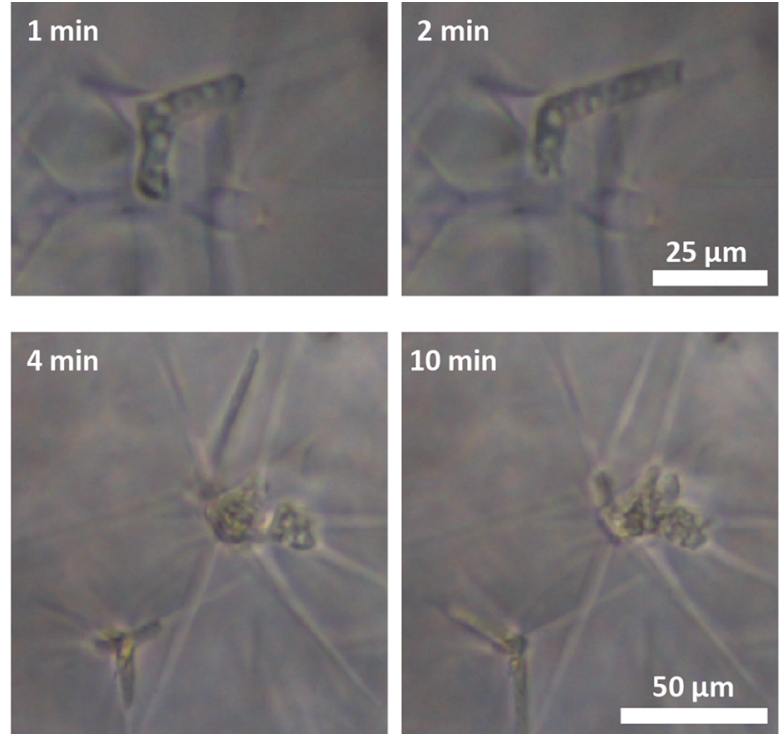

Figure 5. (A) Phase contrast images of A. castellanii moving through the microchannels inside the PAAm. The circle indicates an Acanthamoeba migrating into a side channel $(\varnothing=6.5 \mu \mathrm{m})$. (B) Several Acanthamoebae are stuck in a microchannel junction (arrow) and an Acanthamoeba is stuck inside a cavity. The Acanthamoeba probes the surrounding channels with its Acanthapodia (rectangle).

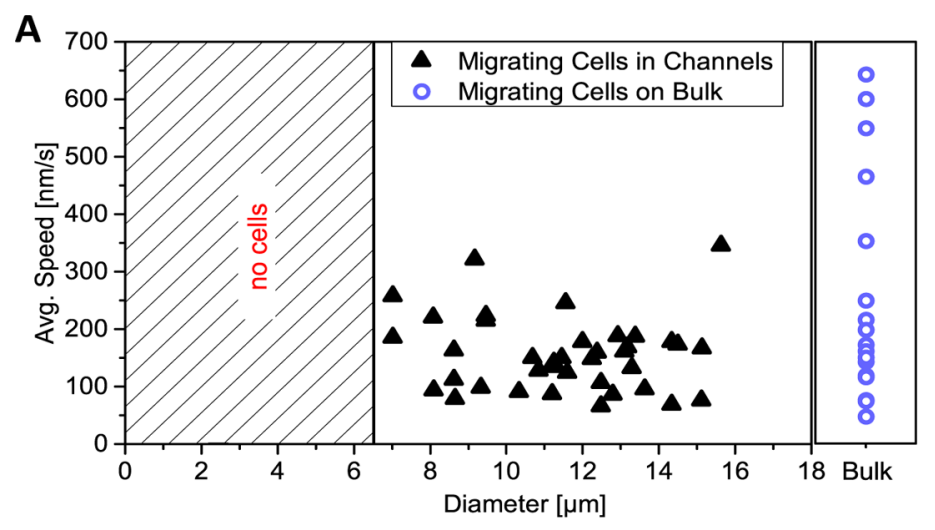

B

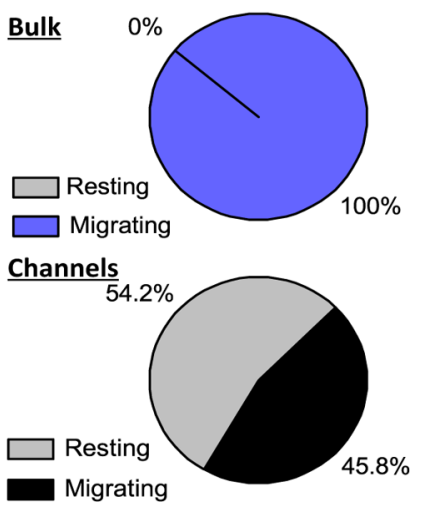

Figure 6. A. castellanii migration inside the microchannels of PAAm. (A) shows the migration speed plotted versus the diameter of the channel the trophozoite was squeezing through. The violet circles on the right represent the migration speeds of $A$. castellanii cells on bulk PAAm samples. It becomes clear that $A$. castellanii trophozoites can squeeze into channels with diameters as small as $6.5 \mu \mathrm{m}$. No cells were observed below that (hatched area). Besides, there is no correlation of cell migration speed with channel diameter, but trophozoites on bulk samples can reach maximum migration speeds almost twice as high as the ones in the microchannels. (B) Percentages of resting and moving A. castellanii trophozoites on bulk samples (total number $N=18)$ and in the microchannel network in our PAAm sample $(N=83)$. Although all amoebae were in motion on bulk samples, $54.2 \%$ of the cells in the microchannel-containing materials were resting in cavities at the junctions of several microchannels.

In general, the fluorescent imaging of the channels is very useful to gain a quick overview of the networks' properties to optimize production parameters for specific experimental requirements. The fluorescent dextrane was chosen such that it entered the microchannels, but not the intrinsic pores of the hydrogel. This strategy can also be used to characterize the sample on many different positions and hence to apply statistical descriptions of global network properties or they can be directly taken at a specific region of interest to gain exact knowledge about sample characteristics at this region. However, it is very useful and accurate to determine the channel diameter of interest in situ during cell experiments by evaluating the cell diameter perpendicular to cellular motion while the cell is migrating through this channel. As the cells need to be optically observed anyway, this does not impose a lot of extra work, but results in the most reliable and quick determination of the relevant channel diameters during cell experiments. This approach overcomes potential issues originating from not having channels with one distinct diameter in the sample, but rather turns this fact into an advantage, as the influence of a variety of diameters on cell migration is automatically analyzed with one experiment. Furthermore, this variety of diameters resembles in vivo situations more accurately, as physiological extracellular environments are not homogeneous at the cell scale. ${ }^{31}$ Similar assumptions hold for channel densities and the appropriate channel density can be chosen by simply changing the position at which experiments are carried out.

PAAm was chosen for the hereby presented experiments since hydrogels are very attractive for many biological applications, ${ }^{32}$ as they are soft ${ }^{33}$ and can support nutrient supply due to their intrinsic nanoporosity. ${ }^{34}$ Specifically, PAAm is often used in cell adhesion experiments because its elastic properties can be defined by cross-linker density in a wide and physiologically important range and its surface can easily be functionalized. ${ }^{32}$ 


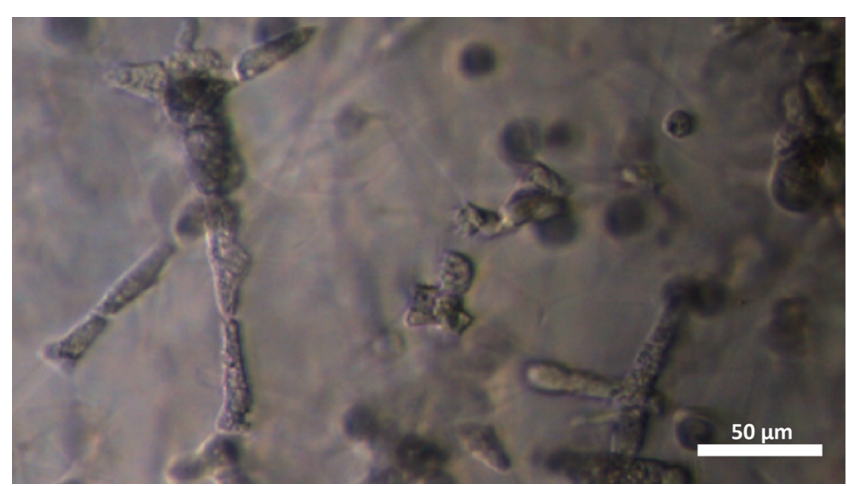

Figure 7. Phase contrast image of densely packed A. castellanii inside the polyacrylamide microchannel network after 1 day of incubation, revealing that the amoebae agglomerate as trophozoites in our substrate on longer time scales.

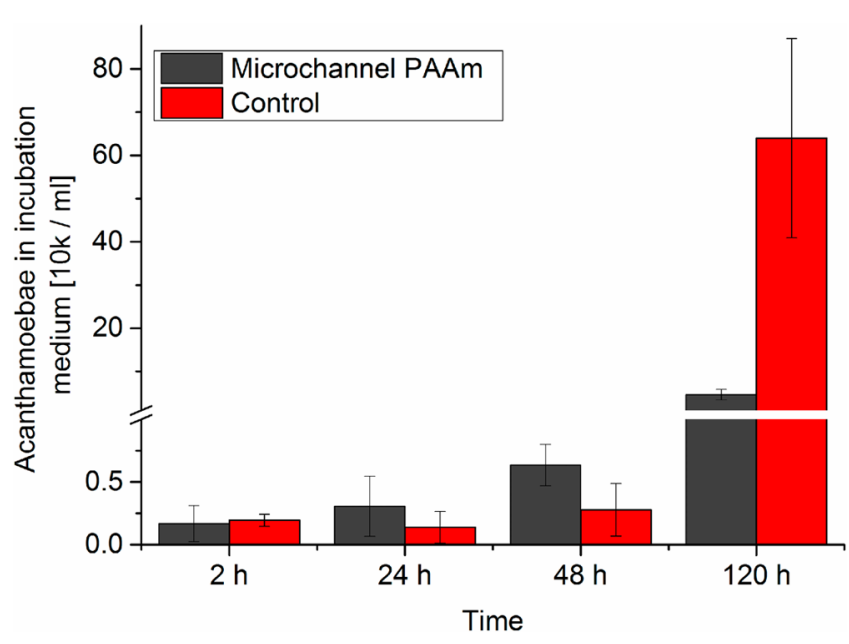

Figure 8. Number of $A$. castellanii in the medium after incubation with a microchannel-containing PAAm sample (black) or an empty cell culture dish (red) shows that although initially more trophozoites remain in the medium when incubated with the microstructured PAAm substrate, this changes strongly after $120 \mathrm{~h}$ incubation time, indicating the potential of our new substrate to remove amoebae from liquids.

The Young's modulus of the polyacrylamide produced in these experiments was approximately $19 \mathrm{kPa}$ (Supporting Information, Table S1), thus being in the range of the matrix elasticity that ensures A. castellanii adhesion. ${ }^{35}$

In general, the fabrication of mechanically robust $3 \mathrm{D}$ materials with well-defined porosities, pore shape and pore organization to control cell migration is highly desirable. However, achieving sufficient mechanical strength, and simultaneously adjustable porosities as well as interconnectivity in soft $3 \mathrm{D}$ architectured materials is a very challenging task. Fabrication of such materials requires complicated processing steps involving high costs. ${ }^{36-38}$ In addition, micrometer-sized pores are often introduced into bulk materials by applying conventional salt-leaching. ${ }^{39,40}$ The pores generated by these conventional methods typically have diameters of several tens of micrometers, and are inverse-opal shaped so that many cells can enrich within a single pore. ${ }^{41}$ Hence, cells in such large pores rather adhere on curved 2D surfaces without being completely surrounded by the material, as it is the case in our microchannels. Furthermore, the materials fabricated by salt-based pore-leaching require large pore fractions in order to guarantee pore interconnectivity, which imposes a large structural limitation. Gas foaming techniques lack the control of pore interconnectivity and often pose problems in the form of processing residuals in the material. ${ }^{42}$ Such limitations are overcome by our novel approach, because we can produce microchannels with high interconnectivity and diameters of down to only a few micrometers because of the special microstructure of the sacrificial ceramic template. Furthermore, our approach can easily be upscaled to fabricate large amounts of microstructured materials, which is challenging for many other techniques such as $3 \mathrm{D}$ printing or cryogelation. ${ }^{42}$

It is also very interesting that most of recent advancements in microporous hydrogel concentrate on anisotropy of the pore structures or increasing the mechanical strength of structured hydrogels. ${ }^{42}$ Our approach concentrates on the creation of hydrogels, which cells can enter freely and in which they can migrate and proliferate without exiting the sample, so that we can study them on long time scales. Incubating A. castellanii cells on our new microchannel-containing PAAm revealed not only that the cells willingly enter and migrate inside the microchannels (Figure 5A), even with diameters much smaller than the cell diameter, but also that the maximum migration speed inside the channels is slower than on conventional 2D substrates (Figure 6A) and, most strikingly, that $A$. castellanii even cease migrating when reaching cavities that had formed at microchannel intersections within the network (Figure 5B). The latter is particularly interesting, because Acanthamoebae are always in motion on the surface of bulk polyacrylamide samples, whereas more than $50 \%$ of the amoeba in microchannel-containing materials were resting during our experiments (Figure 6B). This result is in agreement with a previous study that has reported a decrease in cell migration with increasing pore size. ${ }^{43}$ Interestingly, cancer cells on the other hand have been found to increase their migration speed in narrow channels, ${ }^{44}$ indicating the change of cell migration speed in refined spaces to be a complex phenomenon, which is worth studying. It is noteworthy that in our experiments cells accumulate in much smaller pores compared to the large pores between 96 and 151 $\mu \mathrm{m}$ in size used by Harley et al., ${ }^{43}$ demonstrating that the effect of cell accumulation is also present for small pores. In other words, the parasites were captured by the microchannel network structure of our material, which is highly interesting as this indicates that implementing these structures into, for example, conventional contact lens materials, whose high water contents particularly favor $A$. castellanii growth and hence pose a high infection risk on contact lens users, ${ }^{20,21}$ would significantly decrease this infection risk by keeping the parasites inside the material, where they cannot reach the corneal tissue. It is also important to note here that the trophozoites did not encyst, hence they might be removed without the formation of cysts, which would make them resistant against standard disinfection treatments. $^{45}$

Despite having a cell diameter of $12-35 \mu \mathrm{m},{ }^{12}$ A. castellanii trophozoites were able to migrate through microchannels with diameters down to $6.5 \mu \mathrm{m}$ (Figure 6A). The existence of this lower limit to diameters that $A$. castellanii can squeeze into is reminiscent of that of cells in collagen networks, where cell motion is stopped, if collagen fibers are too dense. ${ }^{9}$ Previous studies on mammalian cells have shown that the nuclear deformability is a critical parameter in $3 \mathrm{D}$ cell migration. ${ }^{8}$ The nucleus of $A$. castellanii trophozoites is one-sixth of the cell diameter with approximately $4.5-6.7 \mu \mathrm{m}$ in size; ${ }^{46}$ this can explain the abrupt absence of migrating cells in channels below approximately $6.5 \mu \mathrm{m}$ in diameter. Another study has shown that 
cancer cell migration is impeded in channels with diameters below $7 \mu \mathrm{m}$, which is in agreement with our results. ${ }^{44}$

Another very striking observation we made was that the trophozoites entering and remaining in the hydrogel leads to a decreased amount of $A$. castellanii in the incubation medium, which means that our new microchannel PAAm can be applied to remove these amoebae from water reservoirs. Since the presence of $A$. castellanii in such reservoirs also enhances the invasiveness of Legionella pneumophila, ${ }^{12}$ this is high of general medical relevance. In contrast to other approaches to remove pathogenic microorganisms from solutions, which are mainly based on chemical capturing ${ }^{47}$ and magnetic capturing, ${ }^{48}$ our approach is solely based on the structural features of the porous hydrogel. This is a clear advantage because the structure is stable, the material is nondegradable and it can be cheaply and efficiently fabricated in high-throughput mode.

\section{CONCLUSION}

In conclusion, our strategy of using porous ceramic templates to incorporate interconnected, labyrinth-like structures of microchannels into hydrogels provides novel, easy-to-fabricate 3D scaffolds for controlling and studying cell migration. As demonstrated, the specific organization and size distribution of microchannels in the material even allows to capture $A$. castellanii by structural material cues, providing an innovative high-throughput strategy for preventing Acanthamoeba-born diseases by, for example, avoiding Acanthamoeba contaminations in contact lens storage cases or water reservoirs. In addition to capturing pathogenic microorganisms, future applications in tissue engineering are highly promising, as in that case the large surface fraction of the cell-material contact in the microchannels can be exploited for controlling cell functions that are induced by this cell-material contact.

\section{ASSOCIATED CONTENT}

\section{S Supporting Information}

The Supporting Information is available free of charge on the ACS Publications website at DOI: 10.1021/acsbiomaterials.8b01009.

Description and results of experiments to characterize the elastic properties as well as the swelling behavior of microchannel-containing PAAm samples; more fluorescent images are presented, which visualize the optical characterization of microchannel network properties (PDF)

Movie S1, time array of phase contrast images with a total duration of $16 \mathrm{~h}$ of $A$. castellanii trophozoites migrating inside the microchannels of a microstructured PAAm substrate produced with the presented technique (AVI)

\section{AUTHOR INFORMATION}

\section{Corresponding Author}

*E-mail: cse@tf.uni-kiel.de.

\section{ORCID $\odot$}

Yogendra Kumar Mishra: 0000-0002-8786-9379 Christine Selhuber-Unkel: 0000-0002-5051-4822

\section{Notes}

The authors declare no competing financial interest.

\section{ACKNOWLEDGMENTS}

We acknowledge funding from the Deutsche Forschungsgemeinschaft (DFG) through the GRK 2154 and the SFB 1261, as well as from the ARVO Foundation (Vistakon Contact Lens Research Fellowship), and the European Research Council (Proof of Concept Grant 768740, Starting Grant 336104). R. A. thanks the DFG for financial support under the Scheme $A D$ 183/17-1. We thank Matthias Leippe for providing $A$. castellannii, Supattra Paveenkittiporn for support with cell migration experiments and data acquisition, Katharina Göpfert for preparing porous substrates, Yannic Hallier for $\mathrm{ZnO}$ tetrapod characterization, and Manuela Lieb for support in the lab.

\section{ABBREVIATIONS}

PAAm polyacrylamide

$\mathrm{t}-\mathrm{ZnO}$ zinc oxide tetrapods

pyg Peptone Yeast Glucose

\section{REFERENCES}

(1) Doyle, A. D.; Carvajal, N.; Jin, A.; Matsumoto, K.; Yamada, K. M. Local 3D matrix microenvironment regulates cell migration through spatiotemporal dynamics of contractility-dependent adhesions. Nat. Commun. 2015, 6, 8720 .

(2) van Helvert, S.; Storm, C.; Friedl, P. Mechanoreciprocity in cell migration. Nat. Cell Biol. 2018, 20 (1), 8-20.

(3) Leach, J. K.; Whitehead, J. Materials-Directed Differentiation of Mesenchymal Stem Cells for Tissue Engineering and Regeneration. ACS Biomater. Sci. Eng. 2018, 4 (4), 1115-1127.

(4) Chang, S. S.; Guo, W.-h.; Kim, Y.; Wang, Y.-L. Guidance of Cell Migration by Substrate Dimension. Biophys. J. 2013, 104 (2), 313-321.

(5) Tayalia, P.; Mendonca, C. R.; Baldacchini, T.; Mooney, D. J.; Mazur, E. 3D Cell-Migration Studies using Two-Photon Engineered Polymer Scaffolds. Adv. Mater. 2008, 20 (23), 4494-4498.

(6) Chaudhuri, O.; Gu, L.; Klumpers, D.; Darnell, M.; Bencherif, S. A.; Weaver, J. C.; Huebsch, N.; Lee, H.-P.; Lippens, E.; Duda, G. N.; Mooney, D. J. Hydrogels with tunable stress relaxation regulate stem cell fate and activity. Nat. Mater. 2016, 15 (3), 326-334.

(7) Discher, D. E.; Janmey, P.; Wang, Y.-L. Tissue Cells Feel and Respond to the Stiffness of Their Substrate. Science 2005, 310 (5751), 1139-1143.

(8) Denais, C. M.; Gilbert, R. M.; Isermann, P.; McGregor, A. L.; te Lindert, M.; Weigelin, B.; Davidson, P. M.; Friedl, P.; Wolf, K.; Lammerding, J. Nuclear envelope rupture and repair during cancer cell migration. Science 2016, 352 (6283), 353.

(9) Wolf, K.; te Lindert, M.; Krause, M.; Alexander, S.; te Riet, J.; Willis, A. L.; Hoffman, R. M.; Figdor, C. G.; Weiss, S. J.; Friedl, P. Physical limits of cell migration: Control by ECM space and nuclear deformation and tuning by proteolysis and traction force. J. Cell Biol. 2013, 201 (7), 1069-1084.

(10) Lautscham, L. A.; Kämmerer, C.; Lange, J. R.; Kolb, T.; Mark, C.; Schilling, A.; Strissel, P. L.; Strick, R.; Gluth, C.; Rowat, A. C.; Metzner, C.; Fabry, B. Migration in Confined 3D Environments Is Determined by a Combination of Adhesiveness, Nuclear Volume, Contractility, and Cell Stiffness. Biophys. J. 2015, 109 (5), 900-913.

(11) Di Giacomo, R.; Krödel, S.; Maresca, B.; Benzoni, P.; Rusconi, R.; Stocker, R.; Daraio, C. Deployable micro-traps to sequester motile bacteria. Sci. Rep. 2017, 7, 45897.

(12) Khan, N. A. Acanthamoeba: biology and increasing importance in human health. FEMS Microbiology Reviews 2006, 30 (4), 564-595.

(13) Huth, S.; Reverey, J. F.; Leippe, M.; Selhuber-Unkel, C. Adhesion forces and mechanics in mannose-mediated acanthamoeba interactions. PLoS One 2017, 12 (5), No. e0176207.

(14) Preston, T. M.; King, C. A. Amoeboid locomotion of Acanthamoeba castellanii with special reference to cell-substratum interactions. Microbiology 1984, 130 (9), 2317-23.

(15) Moore, M. B. Acanthamoeba Keratitis. Arch. Ophthalmol. 1988, 106 (9), 1181-1183. 
(16) Seal, D.; Stapleton, F.; Dart, J. Possible environmental sources of Acanthamoeba spp in contact lens wearers. Br. J. Ophthalmol. 1992, 76 (7), 424-427.

(17) Marciano-Cabral, F.; Cabral, G. Acanthamoeba spp. as Agents of Disease in Humans. Clin. Microbiol. Rev. 2003, 16 (2), 273-307.

(18) Thomas, V.; McDonnell, G.; Denyer, S. P.; Maillard, J.-Y. Freeliving amoebae and their intracellular pathogenic microorganisms: risks for water quality. FEMS Microbiology Reviews 2010, 34 (3), 231-259.

(19) Lorenzo-Morales, J.; Khan, N. A.; Walochnik, J. An update on Acanthamoeba keratitis: diagnosis, pathogenesis and treatment. Parasite 2015, 22, 10.

(20) Reverey, J. F.; Fromme, R.; Leippe, M.; Selhuber-Unkel, C. In vitro adhesion of Acanthamoeba castellanii to soft contact lenses depends on water content and disinfection procedure. Cont Lens Anterior Eye 2014, 37 (4), 262-266.

(21) Coulon, C.; Collignon, A.; McDonnell, G.; Thomas, V. Resistance of Acanthamoeba Cysts to Disinfection Treatments Used in Health Care Settings. Journal of Clinical Microbiology 2010, 48 (8), $2689-2697$

(22) Mishra, Y. K.; Kaps, S.; Schuchardt, A.; Paulowicz, I.; Jin, X.; Gedamu, D.; Freitag, S.; Claus, M.; Wille, S.; Kovalev, A.; Gorb, S. N.; Adelung, R. Fabrication of Macroscopically Flexible and Highly Porous 3D Semiconductor Networks from Interpenetrating Nanostructures by a Simple Flame Transport Approach. Part. Part. Syst. Charact. 2013, 30 (9), 775-783.

(23) Mecklenburg, M.; Schuchardt, A.; Mishra, Y. K.; Kaps, S.; Adelung, R.; Lotnyk, A.; Kienle, L.; Schulte, K. Aerographite: Ultra Lightweight, Flexible Nanowall, Carbon Microtube Material with Outstanding Mechanical Performance. Adv. Mater. 2012, 24 (26), 3486-3490.

(24) Schütt, F.; Signetti, S.; Krüger, H.; Röder, S.; Smazna, D.; Kaps, S.; Gorb, S. N.; Mishra, Y. K.; Pugno, N. M.; Adelung, R. Hierarchical self-entangled carbon nanotube tube networks. Nat. Commun. 2017, 8 (1), 1215 .

(25) Mishra, Y. K.; Adelung, R. ZnO tetrapod materials for functional applications. Mater. Today 2018, 21 (6), 631-651.

(26) Rasband, W. S. ImageJ; U.S. National Institutes of Health: Bethesda, MD, 2005; http://rsb.info.nih.gov/ij/ [Online]. .

(27) Paulowicz, I.; Postica, V.; Lupan, O.; Wolff, N.; Shree, S.; Cojocaru, A.; Deng, M.; Mishra, Y. K.; Tiginyanu, I.; Kienle, L.; Adelung, R. Zinc oxide nanotetrapods with four different arm morphologies for versatile nanosensors. Sens. Actuators, B 2018, 262, 425-435.

(28) Schuster, F. L.; Levandowsky, M. Chemosensory Responses of Acanthamoeba castellanii: Visual Analysis of Random Movement and Responses to Chemical Signals. J. Eukaryotic Microbiol. 1996, 43 (2), $150-158$.

(29) Lawrence, M.; Jiang, Y., Porosity, Pore Size Distribution, Microstructure. In Bio-aggregates Based Building Materials: State-of-the-Art Report of the RILEM Technical Committee 236-BBM, Amziane, S.; Collet, F., Eds. Springer: Dordrecht, The Netherlands, 2017; pp 39-71. DOI: 10.1007/978-94-024-1031-0 2.

(30) Peters, A.; Candau, S. J. Kinetics of swelling of polyacrylamide gels. Macromolecules 1986, 19 (7), 1952-1955.

(31) Khoshgoftar, M.; Wilson, W.; Ito, K.; van Donkelaar, C. C. Influence of tissue- and cell-scale extracellular matrix distribution on the mechanical properties of tissue-engineered cartilage. Biomech. Model. Mechanobiol. 2013, 12 (5), 901-913.

(32) Engler, A. J.; Sen, S.; Sweeney, H. L.; Discher, D. E. Matrix Elasticity Directs Stem Cell Lineage Specification. Cell 2006, 126 (4), 677-689.

(33) Hadden, W. J.; Young, J. L.; Holle, A. W.; McFetridge, M. L.; Kim, D. Y.; Wijesinghe, P.; Taylor-Weiner, H.; Wen, J. H.; Lee, A. R.; Bieback, K.; Vo, B.-N.; Sampson, D. D.; Kennedy, B. F.; Spatz, J. P.; Engler, A. J.; Choi, Y. S. Stem cell migration and mechanotransduction on linear stiffness gradient hydrogels. Proc. Natl. Acad. Sci. U. S. A. 2017, 114 (22), 5647
(34) Stellwagen, N. C. Apparent pore size of polyacrylamide gels: Comparison of gels cast and run in Tris-acetate-EDTA and Tris-borateEDTA buffers. Electrophoresis 1998, 19 (10), 1542-1547.

(35) Gutekunst, S. B.; Grabosch, C.; Kovalev, A.; Gorb, S. N.; Selhuber-Unkel, C. Influence of the PDMS substrate stiffness on the adhesion of Acanthamoeba castellanii. Beilstein J. Nanotechnol. 2014, 5, 1393-1398.

(36) Schaedler, T. A.; Jacobsen, A. J.; Torrents, A.; Sorensen, A. E.; Lian, J.; Greer, J. R.; Valdevit, L.; Carter, W. B. Ultralight Metallic Microlattices. Science 2011, 334 (6058), 962-965.

(37) Cheung, K. C.; Gershenfeld, N. Reversibly Assembled Cellular Composite Materials. Science 2013, 341 (6151), 1219-1221.

(38) Compton, B. G.; Lewis, J. A. 3D-Printing of Lightweight Cellular Composites. Adv. Mater. 2014, 26 (34), 5930-5935.

(39) Raic, A.; Rödling, L.; Kalbacher, H.; Lee-Thedieck, C. Biomimetic macroporous PEG hydrogels as 3D scaffolds for the multiplication of human hematopoietic stem and progenitor cells. Biomaterials 2014, 35 (3), 929-940.

(40) Ganji, Y.; Li, Q.; Quabius, E. S.; Böttner, M.; Selhuber-Unkel, C.; Kasra, M. Cardiomyocyte behavior on biodegradable polyurethane/ gold nanocomposite scaffolds under electrical stimulation. Mater. Sci. Eng., C 2016, 59, 10-18.

(41) Kim, J.; Bencherif, S. A.; Li, W. A.; Mooney, D. J. Cell-Friendly Inverse Opal-Like Hydrogels for a Spatially Separated Co-Culture System. Macromol. Rapid Commun. 2014, 35 (18), 1578-1586.

(42) De France, K. J.; Xu, F.; Hoare, T. Structured Macroporous Hydrogels: Progress, Challenges, and Opportunities. Adv. Healthcare Mater. 2018, 7 (1), 1700927.

(43) Harley, B. A.; Kim, H. D.; Zaman, M. H.; Yannas, I. V.; Lauffenburger, D. A.; Gibson, L. J. Microarchitecture of threedimensional scaffolds influences cell migration behavior via junction interactions. Biophys. J. 2008, 95 (8), 4013-4024.

(44) Rolli, C. G.; Seufferlein, T.; Kemkemer, R.; Spatz, J. P. Impact of Tumor Cell Cytoskeleton Organization on Invasiveness and Migration: A Microchannel-Based Approach. PLoS One 2010, 5 (1), No. e8726.

(45) Johnston, S. P.; Sriram, R.; Qvarnstrom, Y.; Roy, S.; Verani, J.; Yoder, J.; Lorick, S.; Roberts, J.; Beach, M. J.; Visvesvara, G. Resistance of Acanthamoeba Cysts to Disinfection in Multiple Contact Lens Solutions. Journal of Clinical Microbiology 2009, 47 (7), 2040-2045.

(46) Khan, N. Acanthamoeba: Biology and Pathogenesis; Calster Academic Press: 2009

(47) Li, J.; Qi, C.; Lian, Z.; Han, Q.; Wang, X.; Cai, S.; Yang, R.; Wang, C. Cell-Capture and Release Platform Based on Peptide-AptamerModified Nanowires. ACS Appl. Mater. Interfaces 2016, 8 (4), 25112516.

(48) Liu, T.-Y.; Chen, C.-L.; Lee, Y.-C.; Chan, T.-Y.; Wang, Y.-L.; Lin, J.-J. First Observation of Physically Capturing and Maneuvering Bacteria using Magnetic Clays. ACS Appl. Mater. Interfaces 2016, 8 (1), 411-418. 